\title{
New possibilities for the gauging of chiral bosons
}

\author{
E. M. C. Abreu* \\ UNIFEI, Av. BPS 1303, Pinheirinho, 37500-903, Itajubá, MG, Brazil \\ and FEG/UNESP, P.O. Box 205, 12516-410, Guaratinguetá, SP, Brazil \\ A. de Souza Dutra ${ }^{\dagger}$ \\ FEG/UNESP, P.O. Box 205, 12516-410, Guaratinguetá, SP, Brazil \\ C. Wotzasek ${ }^{\ddagger}$ \\ FEG/UNESP, P.O. Box 205, 12516-410, Guaratinguetá, SP, Brazil \\ and IF, UFRJ, P.O. Box 68528, 21945-970, Rio de Janeiro, RJ, Brazil
}

(Received 25 October 2002; published 27 February 2003)

\begin{abstract}
We study a new mechanism for the electromagnetic gauging of chiral bosons showing that new possibilities emerge for the interacting theory of chiral scalars. We introduce a chirally coupled gauge field necessary to mod out the degree of freedom that obstructs gauge invariance in a system of two opposite chiral bosons soldering them together.

DOI: 10.1103/PhysRevD.67.047701

PACS number(s): 11.10.Ef, 04.65.+e
\end{abstract}

In the last few years there has been a great amount of investigation into the proper way to gauge $2 \mathrm{D}$ self-dual fields $[1-7]$ and its $d$-dimensional extension [8,9]. Despite the successes of these indirect gauging schemes for the FloreaniniJackiw (FJ) model, the results reported in [2] show clearly that the explicitly covariant model for chiral bosons put forward by Siegel [1] also suffers from the same difficulties regarding the coupling to gauge fields. In this work we show how the results already reported in the literature can be obtained directly from first principles using the compatibility between field equations and chiral constraints as a guiding rule. We also show that, once this rule is relaxed, new and interesting possibilities emerge. These new gauging rules are the main result of this paper.

We will show the incompatibility between the gauge invariant constraint and the field equations. Then we introduce a gauge field, chirally coupled to chiral matter, which is necessary in order to solder [10] together a right and a left chiral boson. Our main result follows, where we use the dual projection scheme, proposed in $[11,12]$, to study new gauging schemes. Last, final discussions and perspectives are described.

In the literature about this subject, the basic difficulty in chiral boson gauging is pointed out to be the loss of Lorentz covariance which refrains us from using the minimal substitution scheme. It is in fact easy to see that there is an incompatibility between the chiral constraint and the matter field equation. For instance, the action for a flat space-time free scalar field in the light-front coordinates [our notation: $x^{ \pm}$ $\left.=(1 / \sqrt{2})\left(x^{0} \pm x^{1}\right), \quad \epsilon^{+-}=\epsilon^{-+}=1\right]$ is $\mathcal{L}=\partial_{+} \phi \partial_{-} \phi$. The chiral constraint $\partial_{-} \phi \approx 0$ is consistent with the equations of motion (EM) before gauging, but not after. Indeed, suppose

\footnotetext{
*Present address: DCP, CBPF, Rua Xavier Sigaud 150, 22290180, Urca, RJ, Brazil. Email address: everton@cbpf.br

${ }^{\dagger}$ Email address: dutra@feg.unesp.br

‡Email address: clovis@if.ufrj.br and wotzasek@feg.unesp.br
}

we gauge the system through the chiral derivative substitution rule $\partial_{+} \phi \rightarrow \partial_{+} \phi$ and $\partial_{-} \phi \rightarrow D_{-} \phi$ where $D_{ \pm} \phi=\partial_{ \pm} \phi$ $+A_{ \pm}$. The EM for the scalar field now reads $\partial_{+} D_{-} \phi \sim E$ with $E$ being the electric field on the line. Notice that the imposition of the gauge invariant constraint $\left(D_{-} \phi \approx 0\right)$ becomes inconsistent with the field equation due to the appearance of the gauge anomaly. Suppose that the equations of motion for some general chiral theory reads, before gauging, $L \partial_{-} \phi=0$, with $L$ being the differential operator convenient for the Euler-Lagrange matter equation. If the gauging procedure chosen is, for instance, the direct substitution of partial derivatives by their covariant counterparts, the outcome after gauging will read $L D_{-} \phi \sim E$, displaying the abovementioned inconsistency. For chiral bosons, the question one may ask is if there exists any gauging procedure in which the chiral constraint remains compatible both before and after turning on the interactions. As a matter of fact, by examining the Lagrangian density proposed in $[6,7], \mathcal{L}=\partial_{+} \phi \partial_{-} \phi$ $-\partial_{-} \phi \partial_{-} \phi+2 e\left(\partial_{+} \phi-\partial_{-} \phi\right) A_{-}+$gauge terms, we get, as the EM for the matter field, $\partial_{1} D_{-}^{e} \phi=0$ (where $D_{+}^{e} \phi=\partial_{+}$ $\left.+e A_{ \pm}\right)$, and this equation shows that the imposition of a gauge invariant chiral constraint is not obstructed by the gauge anomaly. To obtain this result from first principles we need to find out what the direct gauging scheme leading to this action is. This we do next using the iterative Noether approach. To keep the most generality in the formulation of the problem, we consider the case of a scalar field minimally coupled to a background gravitational field.

The action for the standard minimal coupling of a scalar field to a metric $g_{\mu \nu}$ is

$$
\mathcal{L}_{0}=\frac{1}{2} \sqrt{-g} g^{\mu \nu} \partial_{\mu} \phi \partial_{\nu} \phi
$$

A convenient parametrization of the metric is given by (observe that it does not correspond to a partial gauge fixing but it is consequence of the Weyl symmetry) 


$$
\frac{1}{2} \sqrt{-g} g^{\mu \nu}=\lambda\left(\begin{array}{cc}
2 \lambda_{--} & 1+\lambda_{++} \lambda_{--} \\
1+\lambda_{++} \lambda_{--} & 2 \lambda_{++}
\end{array}\right),
$$

where $\lambda=\left(1-\lambda_{++} \lambda_{--}\right)^{-1}$. For definiteness, from now on, let us consider the case of left chiral models. The (left) FJ and Siegel models can be obtained simply by truncation of this metric as $\lambda_{--}=0$ for Siegel and $\lambda_{--}=0, \lambda_{++}=-1$ for FJ, and this will be called the chiral limit. By computing the EM for the $\phi$ field we observe that (before gauging) the chiral constraint cannot be imposed compatibly for an arbitrary metric's components, differently from what happens in the flat space-time case. However, if we restrict ourselves to the (left) chiral model limit above, where $\lambda_{--}=0$, then we have compatibility for the free theory ( $L \partial_{-} \phi=0$ above) with the chiral constraint $\partial_{-} \phi=0$. This is easily seen if we examine more closely the EM of Eq. (1) before gauging, $\quad 0=\partial_{+}\left\{\lambda\left[2 \lambda_{--} \partial_{+} \phi+\left(1+\lambda_{++} \lambda_{--}\right) \partial_{-} \phi\right]\right\}$ $+\partial_{-}\left\{\lambda\left[2 \lambda_{++} \partial_{-} \phi+\left(1+\lambda_{++} \lambda_{--}\right) \partial_{+} \phi\right]\right\}$. The chiral constraint is not compatible with the EM. However, the restriction to the chiral limit gives $0=\left(\partial_{+}+\partial_{-} \lambda_{++}\right.$ $\left.+\lambda_{++} \partial_{-}\right) \partial_{-} \phi$ for Siegel's EM and $0=\left(\partial_{+}-\partial_{-}\right) \partial_{-} \phi$ for FJ's EM, which shows compatibility with $\partial_{-} \phi \approx 0$. After the Noether gauging with (left) chiral currents (see below), the result is $0=\left(\partial_{+}+\partial_{-} \lambda_{++}+\lambda_{++} \partial_{-}\right) D_{-} \phi$ for Siegel's EM and $0=\left(\partial_{+}-\partial_{-}\right) D_{-} \phi$ for FJ's EM. We see that for (left) chiral couplings, the gauge invariant constraint can be imposed over the field equations without being obstructed by the gauge anomaly. This is the only consistent possibility for the (left) chiral boson.

To implement the Noether procedure we need to compute the conserved currents associated to the global symmetries of the model. The axial-vector current for Eq. (3) is $J_{(A)}^{+}$ $=\lambda\left[2 \lambda_{--} \partial_{+} \phi+\left(1+\lambda_{++} \lambda_{--}\right) \partial_{-} \phi\right]$ and $J_{(A)}^{-}$ $=\lambda\left[2 \lambda_{++} \partial_{-} \phi+\left(1+\lambda_{++} \lambda_{--}\right) \partial_{+} \phi\right]$. Defining the vector current as dual to the axial-vector current is not really a restriction of our method since this is a feature of two dimensions. In any case, one can show that in the non-Abelian case, where the vector current can be defined as a Noether current, everything works as discussed here. Hence, the vector current is defined as dual to the axial one, $J_{(V)}^{\mu}=* J_{(A)}^{\mu}$, where the usual Hodge transformation must be generalized to $* J_{(A)}^{\mu}=\sqrt{-g} g^{\mu \nu} \epsilon_{\nu \lambda} J_{(A)}^{\lambda}$ in order to take into account the presence of the gravitational background. A simple calculation shows that $J_{(V)}^{+}=-\partial_{-} \phi$ and $J_{(V)}^{-}=\partial_{+} \phi$ are topologically conserved, as they should be. Observe that the vector current is metric independent, being the same for the chiral models defined by truncation of the metric.

Having the axial-vector and vectorial currents in hand, we are now in position to compute the right and left chiral currents. We find $J_{(L)}^{+}=2 \lambda_{--} \lambda\left(\lambda_{++} \partial_{-} \phi+\partial_{+} \phi\right)$ and $J_{(L)}^{-}$ $=2 \lambda\left(\lambda_{++} \partial_{-} \phi+\partial_{+} \phi\right)$ for the left current and $J_{(R)}^{-}$ $=2 \lambda_{++} \lambda\left(\lambda_{--} \partial_{+} \phi+\partial_{-} \phi\right) \quad$ and $J_{(R)}^{+}=2 \lambda\left(\lambda_{--} \partial_{+} \phi\right.$ $\left.+\partial_{-} \phi\right)$ for the right current. Observe that the (left) chiral boson limit $\left(\lambda_{--}=0\right)$ destroys the $J_{(L)}^{+}$component, leaving the left current holomorphically conserved, while the right chiral current has both components nonvanishing. This is certainly a desired result for the chiral case. In fact, in the flat space-time theory for the free scalar field, there are two sepa- rated affine invariances for the left and right chiral sectors. These symmetries are reflected by the fact that both the right and left chiral currents have only one nonzero component $J_{(L)}^{-}=J_{(L)}^{-}\left(x^{+}\right)$and $J_{(R)}^{+}=J_{(R)}^{+}\left(x^{-}\right)$, since $\partial_{-} J_{(L)}^{-}=0$ and $\partial_{+} J_{(R)}^{+}=0$ and generate two commuting affine algebras. However, in the chiral case, only one of these currents keeps this property, i.e., either $J_{(L)}^{-}=J_{(L)}^{-}\left(x^{+}\right)$but $J_{(R)}^{+} \neq J_{(R)}^{+}\left(x^{-}\right)$ or $J_{(L)}^{-} \neq J_{(L)}^{-}\left(x^{+}\right)$but $J_{(R)}^{+}=J_{(R)}^{+}\left(x^{-}\right)$. This can also be seen from the fact that, for chiral theories, while the vector and axial-vector transformations are global symmetries, the affine transformations are semilocal symmetries. Take, for instance, the case of a left Siegel boson. The semilocal shift $\phi \rightarrow \phi+\alpha\left(x^{+}\right)$certainly leaves the action invariant, but $\phi$ $\rightarrow \phi+\alpha\left(x^{-}\right)$does not. The Noether current is immediately identified as $J^{-}=2\left(\partial_{+} \phi+\lambda_{++} \partial_{-} \phi\right)$ and $J^{+}=0$ which is easily seen to be the (left) chiral limit of $J_{(L)}^{+}$and $J_{(L)}^{-}$above.

We shall examine next the coupling of all these four currents with an external electromagnetic field. We do this iteratively, introducing the necessary Noether counterterms. Let us first examine the coupling with the vector current $J_{(V)}^{+}$and $J_{(V)}^{-}$in the standard way: $\mathcal{L}_{0} \rightarrow \mathcal{L}_{1}=\mathcal{L}_{0}+A_{+} J_{(V)}^{+}+A_{-} J_{(V)}^{-}$, where $\mathcal{L}_{0}$ is defined by Eq. (1). The global vector symmetry present in $\mathcal{L}_{0}$ has been lifted to a local symmetry in $\mathcal{L}_{1}$. We observe that, after gauging, the vector current remains conserved, but the axial-vector current does not. A direct calculation shows that $\partial_{\mu} J_{(A)}^{\mu}=\partial_{-} A_{+}-\partial_{+} A_{-}=E$, which, again, being independent of the metric elements, is valid for all cases. By computing the EM for $\mathcal{L}_{1}$ we clearly see that the original compatibility between the constraint and EM has been destroyed for gauging with vector currents $J_{(V)}^{+}$and $J_{(V)}^{-}$ due to the presence of the anomaly. Therefore, we have to rule out vector current gauging as inappropriate for chiral theories.

Let us consider next the case of axial-vector coupling. The free action changes to $\mathcal{L}_{0} \rightarrow \mathcal{L}_{1}=\mathcal{L}_{0}+A_{+} J_{(A)}^{+}$ $+A_{-} J_{(A)}^{-}$. Differently from the vector case, under an axialvector transformation the action $\mathcal{L}_{1}$ does not remain invariant but its variation is given by $\delta \mathcal{L}_{1}=-\delta\left\{\lambda\left[\lambda_{--} A_{+}^{2}\right.\right.$ $\left.\left.+\lambda_{++} A_{-}^{2}+1+\lambda_{++} \lambda_{--} A_{+} A_{-}\right]\right\}$. A further modification of the action as

$$
\begin{aligned}
\mathcal{L}_{1} \rightarrow \mathcal{L}_{2}= & \mathcal{L}_{1}+\frac{1}{1-\lambda_{++} \lambda_{--}}\left[\lambda_{--} A_{+}^{2}+\lambda_{++} A_{-}^{2}\right. \\
& \left.+\left(1+\lambda_{++} \lambda_{--}\right) A_{+} A_{-}\right]
\end{aligned}
$$

leaves the action $\mathcal{L}_{2}$ invariant under an axial-vector transformation. It is simple to check that here the axial-vector current remains conserved, while the gauge coupling modifies the vector current, which now fails to be conserved. Finally, by checking the EM, one notices the incompatibility of the gauged constraint with the field equation, which also rules out this coupling as inappropriate for the chiral models.

We are then left only with the possibilities of chiral current couplings. We have explicitly checked that for left chiral bosons, the coupling with the right chiral current results incompatible. Now, let us work out explicitly the coupling of the left chiral current and verify that this is the only possible 
consistent way of coupling. The Noether procedure then gives $\mathcal{L}_{0} \rightarrow \mathcal{L}_{1}=\mathcal{L}_{0}+A_{+} J_{(L)}^{+}+A_{-} J_{(L)}^{-}$whose variation reads $\delta \mathcal{L}_{1}=\lambda\left[\left(2 \lambda_{++} \lambda_{--} A_{+} \partial_{-} \alpha+2 A_{-} \partial_{+} \alpha\right)-\delta\left(\lambda_{--} A_{+}^{2}\right.\right.$

$\left.\left.+\lambda_{++} A_{-}^{2}\right)\right]$. The second term can be reabsorbed into a redefinition of the action as

$$
\mathcal{L}_{1} \rightarrow \mathcal{L}_{2}=\mathcal{L}_{1}+\lambda_{++} A_{-}^{2}+\lambda_{--} A_{+}^{2},
$$

but the first piece cannot be eliminated by any choice of a Noether counterterm. This is true even for the truncated chiral limit but it is hardly a surprise since the gauged action for chiral models is not expected to be gauge invariant. However, this action has the nice property of having its variance independent of the matter fields.

The important point to observe is that the truncation process used to go from the nonchiral to the chiral case does not change the nature of the coupling, which means that the vector, axial-vector, and chiral couplings studied above remain the same after truncation. This is certainly different from the projection process using chiral constraints that transform, for instance, the vector coupling into a chiral coupling.

It must be pointed out that the FJ chiral limit of $\mathcal{L}_{2}$ in Eq. (4) is identical to that in Refs. [6,7], obtained via left projection of the chiral Schwinger model (SM), but the other limits here are new results. In particular we emphasize that we did not invoke the chiral SM at any level, but derive our result from first principles.

Finally, we believe that for chiral boson actions which use infinite auxiliary fields [5] (the equivalence between both proposals was demonstrated in Ref. [13]), the procedure is the same, but now we have a sum of infinite chiral currents, one for each field, which are embedded in an external electromagnetic field. Similarly, for the Paiti-Sorokin-Tonin (PST) formulation [14] of the Siegel chiral boson, it is not difficult to see that that procedure also works.

As a simple illustration of the use of the gauged theory, the property mentioned above is explored now in order to solder together two bosons of opposite chiralities. Details can be found in Ref. [10]. The soldering process is nontrivial since the simple sum of the actions of a right and a left chiral boson is not equal to the action of a single scalar field.

For computational convenience we use front-form variables. In this coordinate system, the action for left and right FJ chiral bosons reads, respectively $\mathcal{L}_{0}^{( \pm)}=\mp \dot{\phi}_{ \pm} \phi_{ \pm}^{\prime}$ $-\left(\phi_{ \pm}^{\prime 2}\right)$, where the overdot and prime have their usual significance as time and space derivatives, respectively. We know, from their field equations, that these models have a residual invariance under a semilocal transformation $\phi_{ \pm}$ $\rightarrow \widetilde{\phi}_{ \pm}=\phi_{ \pm}+\alpha_{ \pm}(t)$. Therefore, if one defines a scalar field as a combination of these chiral ones as $\Phi=\phi_{+}-\phi_{-}$, then clearly the combination of the two semilocal transformations above will not lead to a vector transformation for the scalar field, unless some constraint is imposed over each individual component. This is the role played by the gauge field. We can then follow the gauging procedure described above to obtain the action of an interacting chiral boson coupled to a gauge field through their chiral left and right currents: respectively, $\mathcal{L}_{0}^{ \pm} \rightarrow \mathcal{L}_{1}^{ \pm}=\mathcal{L}_{0}^{ \pm} \mp 2 A\left(\dot{\phi}_{ \pm} \pm \phi_{ \pm}^{\prime}\right)$. Here $A$ is the space component of the gauge field, i.e., $A$ is the so-called soldering field, an auxiliary field that will be eliminated (integrated) through the field equations (or through the path integral, as below). Although each individual (gauged) action is variant under a gauge transformation, as we have seen in the last section [see Eq. (4)], one can verify that the new action $\mathcal{L}=\mathcal{L}_{1}^{-}+\mathcal{L}_{1}^{+}-2 A^{2}$ is indeed invariant. The last term is a contact term that compensates for the noninvariances of each chirality. Now, we can integrate out the gauge field $A$, as $\exp \left(i \int d^{2} x \mathcal{W}\right)=\int[d A] \exp \left(i \int d^{2} x \mathcal{L}\right)$ to obtain $\mathcal{W}$ $=\frac{1}{2} \partial_{\mu} \Phi \partial^{\mu} \Phi$, which is the action for the scalar field $\Phi$ $=\phi_{+}-\phi_{-}$defined as a combination of a right and a left chiral boson.

In this section we consider another approach to the chiral gauge problem based on the dual projection $[11,12]$ of scalar fields. The basic idea in this scheme is to gauge the scalar theory, using the obvious covariance that it possesses, and then "break" the basic fields, in phase space, into its chiral components. For definiteness, let us consider a well-known generalized chiral SM (GCSM)

$$
\mathcal{L}=\bar{\Psi} \gamma^{\mu}\left(i \partial_{\mu}+e_{R} A_{\mu} \frac{1+\gamma_{5}}{2}+e_{L} A_{\mu} \frac{1-\gamma_{5}}{2}\right) \Psi,
$$

where $\Psi$ is a two-component spinor

$$
\Psi=\left(\begin{array}{c}
\psi_{R} \\
\psi_{L}
\end{array}\right)
$$

and $\psi_{L}$ and $\psi_{R}$ are the Weyl components. Its quantum dynamics is easily computed using the bosonized version after a convenient redefinition of the fields, $\mathcal{L}_{\text {boson }}=1 / 2 \partial_{\mu} \phi \partial^{\mu} \phi$ $-e_{-} A_{\mu} \partial^{\mu} \phi+e_{+} A_{\mu} \epsilon^{\mu \nu} \partial_{\nu} \phi+g^{2} a / 2 A_{\mu} A^{\mu} \quad$ where $e_{R}$ $=1 / 2\left(e_{+}+e_{-}\right), e_{L}=1 / 2\left(e_{+}-e_{-}\right), g^{2}=\left(e_{-}^{2}+e_{+}^{2}\right) / 2$, and $a$ is an arbitrary regularization parameter. The interesting point in working with the GCSM is that one can easily obtain the four versions of the SM considered in the literature: vector $\mathrm{SM}\left(e_{-}=0 \Rightarrow e_{L}=e_{R}=e\right)$, axial $\mathrm{SM}\left(e_{+}=0 \Rightarrow e_{L}=-e_{R}\right.$ $=e)$, right chiral SM $\left(e_{L}=0 \Rightarrow e_{+}=e_{-}=e\right)$, and left chiral $\mathrm{SM}\left(e_{R}=0 \Rightarrow e_{+}=-e_{-}=e\right)$. The dual projection scheme permits one to realize that the second-order differential EM for the matter field coming from the action $\mathcal{L}_{\text {boson }}$ contains both right and left moving solutions. Let us reduce this action to its first-order form as

$$
\begin{aligned}
\mathcal{L}= & \pi \dot{\phi}-\frac{1}{2} \pi^{2}-\frac{1}{2}\left(\phi^{\prime}\right)^{2}-\left(e_{-} A_{0}+e_{+} A_{1}\right) \pi \\
& +\left(e_{-} A_{1}+e_{+} A_{0}\right) \phi^{\prime}+\frac{1}{2} A_{\mu} M^{\mu \nu} A_{\nu},
\end{aligned}
$$

where $A_{\mu} M^{\mu \nu} A_{\nu}=a g^{2} A_{\mu} A^{\mu}-\left(e_{-} A_{0}+e_{+} A_{1}\right)^{2}$ and $\pi$ is the second field mentioned above obtained through Legendre transformation from $\mathcal{L}_{\text {boson }}$. We observe that the fields $\phi$ and $\pi$ cannot be our chiral fields describing, independently, the right and left dynamics since they appeared coupled in the symplectic sector of Eq. (5). We must therefore look for a linear combination of them that diagonalizes the action. If we introduce the new fields $\varphi$ and $\rho$ as an $\mathrm{SO}(2)$ rotation of the $\pi$ and $\phi$ fields as 


$$
\left(\begin{array}{c}
\pi \\
\phi^{\prime}
\end{array}\right)=\left(\begin{array}{cc}
\cos \theta & \sin \theta \\
-\sin \theta & \cos \theta
\end{array}\right)\left(\begin{array}{c}
\varphi^{\prime} \\
\rho^{\prime}
\end{array}\right),
$$

then we quickly realize that, in order to diagonalize the action (5), we must have $\theta=\pi / 4$, and consequently the following canonical transformations (CTs) [15] $\phi^{\prime}=\varphi^{\prime}+\rho^{\prime}$ and $\pi=\varphi^{\prime}-\rho^{\prime}$, which we take as our definition of the chiral fields $\varphi$ and $\rho$. In terms of these new fields the action for the GCSM, $\mathcal{L}_{\text {boson }}$, now reads $\mathcal{L}_{\text {boson }}=\mathcal{L}_{\varphi}+\mathcal{L}_{\rho}+\mathcal{L}_{A}$ where $\mathcal{L}_{\varphi}$ $=\dot{\varphi} \varphi^{\prime}-\varphi^{\prime 2}+2 e_{L} \varphi^{\prime} A_{-}, \mathcal{L}_{\rho}=-\dot{\rho} \rho^{\prime}-\rho^{\prime 2}+2 e_{R} \rho^{\prime} A_{+}$, and $\mathcal{L}_{A}=1 / 2 A_{\mu} M^{\mu \nu} A_{\nu}$ with $A_{ \pm}=A_{0} \pm A_{1}$.

There are many interesting points to observe in this nowdiagonalized action. Notice that in the free case the chiral fields satisfy $\partial_{-} \phi=0$ and $\partial_{+} \rho=0$ so that they are, respectively, left and right chiral modes. The actions for the free components are those proposed by FJ. Also, no matter which model is in discussion, the interaction piece shows that these fields only couple to a proper chiral combination of the electromagnetic field, $A_{-}$and $A_{+}$, respectively, but the coupling constant is, obviously, model dependent. For the vector and axial-vector SMs both chiral components are coupled to the photon field but the chiral SM will leave one chirality free, either $\varphi$ when $e_{L}=0$ or $\rho$ when $e_{R}=0$. In all these cases the mass term will change appropriately. What should be regarded as the most important point is that this dual projection shows the chiral bosons correctly coupled to the photon field as proposed by Refs. [6,7]. However, in our case we got the coupling of both chiralities at once. In the name of completeness, analyzing the case of the chiral bosons models with infinite auxiliary terms coupled to gravity, using the dual projection, it was shown [4] that this model has nonmover fields, the notons (introduced by Hull [16] to cancel the Siegel anomaly), which couple naturally with the gravitational field. For the PST version of the Siegel chiral boson, it was proved that its spectrum is composed by a FJ particle and a PST version of the noton [12].

We are now in position to compare this result with the chiral projection technique adopted in Ref. [7]. From the CTs defined above we get $\pi-\phi^{\prime}=-2 \rho^{\prime}$ and $\pi+\phi^{\prime}=2 \varphi$, so that the chiral constraints $\pi \pm \phi^{\prime}=0$ lead to $\varphi=0$ and $\rho$ $=0$ as constraints, respectively. It should be observed that the chiral component eliminated through this process need not be a free component, so that one does not need to start with a chiral SM in order to get the gauged chiral boson. So except for some very awkward possibilities, any model will do. Finally, we must observe that the CTs above lead to a very sensible interpretation of the chiral bosons $\varphi$ and $\rho$ as being the bosonized components of the Weyl fermions in the GCSM since they are coupled to the gauge field with the same strength as their fermionic counterparts.

In conclusion, in this work we have studied the problem of coupling self-dual scalar fields in 2D to an external electromagnetic field described by a vector potential $A_{\mu}$. We have recognized the basic difficulty as an incompatibility between the gauge invariant chiral constraint and the field equation for the matter field. Using consistency as a guiding rule, we have worked out the coupling of gauge fields with different matter currents and observed that the only consistent coupling for a left chiral matter is with a left chiral current. This explain the results obtained with the use of the chiral projector on the SM. As an application we verified that in order to solder together a left and a right chiral boson into a scalar field, we must (chirally) couple them to a gauge field that will mod out the degree of freedom that obstructs gauge invariance.

This work was partially supported by Fundação de Amparo à Pesquisa do Estado de São Paulo (FAPESP) and Conselho Nacional de Desenvolvimento Científico e Tecnológico $(\mathrm{CNPq}) . \mathrm{CNPq}$ and FAPESP are Brazilian research agencies.
[1] W. Siegel, Nucl. Phys. B238, 307 (1984).

[2] S. J. Gates, Jr. and W. Siegel, Phys. Lett. B 206, 631 (1988).

[3] R. Floreanini and R. Jackiw, Phys. Rev. Lett. 59, 1873 (1987); Y. Frishman and J. Sonnenschein, Nucl. Phys. B301, 346 (1988); J. Sonnenschein, ibid. B309, 752 (1988); D. Bazeia, Mod. Phys. Lett. A 5, 2497 (1990); S. Ghosh and P. Mitra, ibid. 6, 2957 (1991); Phys. Rev. D 44, 1332 (1991); C. Wotzasek, Z. Phys. C 70, 345 (1996); L. S. Brown and R. I. Nepomechie, Phys. Rev. D 35, 3239 (1987); L. Mezincescu and R. I. Nepomechie, ibid. 37, 3067 (1988).

[4] N. R. F. Braga and C. Wotzasek, Mod. Phys. Lett. A 10, 173 (1995).

[5] B. McClain, Y. S. Wu, and F. Yu, Nucl. Phys. B343, 689 (1990); C. Wotzasek, Phys. Rev. Lett. 66, 129 (1991).

[6] S. Bellucci, M. F. L. Golterman, and D. N. Petcher, Nucl. Phys. B326, 307 (1989).

[7] K. Harada, Phys. Rev. Lett. 64, 139 (1990).

[8] E. Bergshoeff, Class. Quantum Grav. 13, 2875 (1996).

[9] J. Schwarz, Phys. Lett. B 395, 191 (1997).

[10] M. Stone, "How to make a bosonized Dirac fermion from two bosonized Weyl fermions," Report No. ILL/23/89; Phys. Rev. Lett. 63, 731 (1989); Nucl. Phys. B327, 399 (1989); D. Depireux, S. J. Gates, Jr., and Q-Han Park, Phys. Lett. B 224, 364 (1989); E. Witten, Commun. Math. Phys. 144, 189 (1992); R. Amorim, A. Das, and C. Wotzasek, Phys. Rev. D 53, 5810 (1996); E. M. C. Abreu, R. Banerjee, and C. Wotzasek, Nucl. Phys. B509, 519 (1998); for a review see C. Wotzasek, "Soldering Formalism: Theory and Applications," hep-th/9806005.

[11] E. M. C. Abreu and C. Wotzasek, Phys. Rev. D 58, 101701 (1998); C. Wotzasek, ibid. 58, 125026 (1998); R. Banerjee and C. Wotzasek, Nucl. Phys. B527, 402 (1998); Phys. Rev. D 63, 045005 (2001).

[12] E. M. C. Abreu, J. High Energy Phys. 11, 008 (2002).

[13] C. Wotzasek, Phys. Rev. D 44, R1623 (1991).

[14] P. Pasti, D. Sorokin, and M. Tonin, Leuven Notes in Mathematical and Theoretical Physics, Series B (Leuven University Press, Leuven, Belgium, 1996), Vol. 6, p. 167; Phys. Rev. D 55, 6292 (1997).

[15] R. Banerjee and S. Ghosh, Phys. Lett. B 482, 302 (2000).

[16] C. M. Hull, Phys. Lett. B 206, 234 (1988); 212, 437 (1988). 